\title{
FSJ-Jahrestagung: Gut begleitet durch das Freiwillige Soziale Jahr ${ }^{1}$
}

\author{
Dr. Jaana Eichhorn \\ Bundestutorat FSJ und BFD im Sport, Deutsche Sportjugend \\ Bundesarbeitskreis FSJ | eichhorn@dsj.de
}

Die besonderen Bildungschancen eines Freiwilligendienstes liegen in der Verbindung aus praktischen Tätigkeiten in der Einsatzstelle und der pädagogischen Begleitung dieses Einsatzes. Freiwillige wollen, können und sollen ihr Freiwilliges Soziales Jahr als ein Lern- und Orientierungsjahr erleben. Damit dies tatsächlich gelingt, ist mehr notwendig als eine gute Seminararbeit. Um die Bildungsarbeit von Trägern und Einsatzstellen außerhalb der Seminare in den Fokus zu nehmen, galt ihr die vom Bundesministerium für Familie, Senioren, Frauen und Jugend (BMFSFJ) finanzierte und gemeinsam mit dem Bundesarbeitskreis Freiwilliges Soziales Jahr (BAK FSJ) vorbereitete Trägertagung 2013.

Freiwillige erhalten im FSJ eine persönliche Begleitung, die sie in ihrer Entwicklung fördert; sie werden durch qualifizierte Mitarbeiterinnen und Mitarbeiter der Träger und Einsatzstellen fachlich angeleitet und bei Fragen und Problemen unterstützt. Diese Aufgaben sind sowohl von Einsatzstellen als auch von Trägern zu leisten. In ihrer Gesamtverantwortung für die Qualität und Durchführung des FSJ sind die Träger zudem gehalten, die Einsatzstellen bei ihrer wichtigen und verantwortungsvollen Arbeit zu unterstützen und zu fördern. Denn es sind zuerst die Einsatzstellen, die tagtäglich unmittelbar mit den jungen Freiwilligen zu tun haben, deren Fähigkeiten und Interessen fördern, Bedürfnisse erkennen, aber auch auf deren Sorgen und Nöte sachkundig und angemessen reagieren müssen. Andererseits haben auch Einsatzstellen und Träger Interessen und Bedürfnisse, die zu berücksichtigen sind, um das FSJ erfolgreich zu gestalten. Ziel der Tagung war es daher, die verschiedenen Elemente der Bildungs- und Begleitungsarbeit außerhalb der Seminare in den Blick zu nehmen. Erstmals wurden neben Trägervertretern und -vertreterinnen auch Einsatzstellen aus dem gesamten Bundesgebiet zur Jahrestagung eingeladen.

In zwei Grundsatzreferaten betrachteten Dr. Theresa Lempp (Technische Universität Dresden) sowie Prof. Dr. Heiner Keupp (ehem. Ludwig-Maximilians-Universität München) Bildung und Begleitung im FSJ aus wissenschaftlicher, insbesondere aber entwicklungspsychologischer Perspektive. Sie zeichneten Freiwillige im

1 Die Tagung fand vom 19. bis 20. November 2013 in Berlin statt. 
FSJ als junge Erwachsene in einer Übergangsphase, einer risikobehafteten, durch Diskontinuitäten gekennzeichneten Zeit. Zentrale Aufgabe der Jugendfreiwilligendienste als Teil der Kinder- und Jugendarbeit ist es insofern, Heranwachsende dazu zu befähigen, jugendspezifische Entwicklungsaufgaben in einer spätmodernen Gesellschaft zu bewältigen. Dabei steht die eigene Identitätsarbeit im Zentrum.

Erforderlich sind neben passenden Rahmenbedingungen auch vielfältige Möglichkeiten, Selbstwirksamkeit zu erfahren. Dies geschieht vor dem Hintergrund einer sich verlängernden Jugendphase, in der die Übernahme realer Verantwortung sowie die materielle Selbständigkeit sich nach hinten verschieben. Verlängert werden zudem die Bildungsphase und der Verbleib in der Familie. Aufgrund der stärkeren Unsicherheiten wird von den Jugendlichen eine zielgerichtete Ausbildung erwartet, ohne ihnen das Vertrauen zu vermitteln, dass sie damit die gewünschte materielle Sicherheit dauerhaft erreichen können. Von den Freiwilligendiensten wird vor diesem Hintergrund die gezielte Vermittlung von Kompetenzen erwartet, eine Stärkung persönlicher Identität, die auf eine bessere Passung in den Arbeitsmarkt zielt - ein Wunsch, der den pädagogischen Vorstellungen der Träger z. T. zuwiderläuft. Gleichzeitig gibt es gegenläufige Tendenzen, den Freiwilligendienst als Moratorium zu sehen, als Auszeit, als Möglichkeit der Entschleunigung.

In der Zeit des Heranwachsens werden, so betonten die Vortragenden, psychosoziale wie qualifikatorische Grundlagen für ein gelingendes Erwachsenwerden gelegt. Die Rahmenbedingungen sind in einer sich dramatisch verändernden Gesellschaft jedoch zunehmend schwierig. Schule und Bildung werden beschleunigt, Entwicklungsaufgaben verdichten sich und „employability“, die Beschäftigungsfähigkeit, scheint zum übergreifenden Ziel zu werden, dem andere Lebensräume untergeordnet sind. Die aktuelle gesellschaftliche Entwicklung weist wenig Spielräume für das Experimentieren mit möglichen Identitätsentwürfen auf, was zu wachsenden psychosozialen Problemen führt. Jugendliche benötigen aber Verwirklichungschancen für ein selbstbestimmtes Leben auch neben dem Mainstream, sie müssen die Möglichkeit erhalten, Um- und Irrwege zu wählen. Dies widerspricht der Erwartung an Kinder und Jugendliche, typische Entwicklungsaufgaben immer schneller zu „erledigen“ - egal, ob es um Körpererfahrung, den Umgang mit Sexualität, den Umbau sozialer Beziehungen, die Identitätsfindung oder den Großbereich Lernen/Bildung/Berufswahl geht.

In der Zeit der Beschleunigung bei gleichzeitig wachsenden Aufgaben nimmt die Relevanz informeller Bildung - wie sie die Freiwilligendienste bestimmt - zu. Studien zeigen immer deutlicher, dass die Zukunftschancen Heranwachsender nicht nur durch formelle, sondern auch durch informelle Bildung beeinflusst werden, etwa in Alltag, Familie, Nachbarschaft, Sport, Freizeit oder Jugendarbeit. 
Besondere Bedeutung kommt dabei der Partizipation zu, die bürgerschaftliches Engagement in ihren unterschiedlichen Ausprägungen typischerweise kennzeichnet. Sie erlaubt die Erfahrung von Selbstwirksamkeit, die als entscheidend für eine erfolgreiche Persönlichkeitsentwicklung eingeschätzt wird. Gerade in einer Zeit, in der „alles schon erfunden ist“, wie Prof. Keupp betonte, in der Kinder und Jugendliche also wenig Chancen auf eine tatsächliche Gestaltung ihres Lebens haben und Bildung allzu häufig durch passive Rezeption charakterisiert ist, werden Gelegenheiten für Beteiligung, Partizipation und Teilhabe unabdingbar: Performanz ist und bleibt die Voraussetzung für Selbst-Erfahrung. Freiwilligendienste bieten eine ideale Grundlage für Selbstwirksamkeitserfahrungen, da sie einen geschützten Rahmen bieten, in dem Jugendliche die Folgen ihres Handelns direkt erleben können. Während der Freiwilligendienst an sich optimale Möglichkeiten zum Selbsttätigwerden bietet, liegt es insbesondere an dem/der AnleiterIn, die Heranwachsenden im Bereich der Selbstorganisation zu unterstützen. Auch viele Seminarangebote bieten Freiwilligen gute Gelegenheiten, sich mit Selbstmanagement und hilfreichen Techniken vertraut zu machen. Insgesamt sind, da waren sich Prof. Keupp und Dr. Lempp einig, Freiwilligendienste als geschützte Bildungsorte sehr hilfreich.

In sieben verschiedenen Arbeitsgruppen - von denen je zwei besucht werden konnten - wurden unterschiedliche Formen der Begleitung außerhalb der Seminararbeit von Einsatzstellen- und TrägervertreterInnen gemeinsam diskutiert. Im Zentrum der Arbeitsgruppe eins (Moderation: Doris Siebolds, Arbeiter-Samariter-Bund) stand die Zusammenarbeit von Trägern und Einsatzstellen, während sich die zweite Arbeitsgruppe (Axel-Kurt Schubert, Bundesamt für Familie und zivilgesellschaftliche Aufgaben, BAFzA) Konfliktmanagement und Krisenintervention, insbesondere zwischen Einsatzstellen und Trägern, widmete. Eine dritte Arbeitsgruppe (Tina Stampfl, Arbeiterwohlfahrt Bundesverband) diskutierte Einsatzstellen als Engagementorte und Bildungspartner und nahm sowohl Erwartungen und Bedürfnisse der Einsatzstellen als auch ihre pädagogischen Aufgaben in den Blick. Arbeitsgruppe vier (Hartmut Brombach, Internationaler Bund) erörterte mit dem Bereich Arbeitsmarktneutralität ein politisch besonders interessantes Thema und zeigte den Spagat zwischen arbeitsmarktneutralem Einsatz einerseits und beruflicher Orientierungsmöglichkeit - die eben mehr umfasst, als die Beschränkung auf Hilfstätigkeiten - andererseits auf.

Während die fünfte Arbeitsgruppe sich dem Incoming im FSJ widmete (Stefan Malik, Bund der Deutschen Katholischen Jugend), betrachtete Arbeitsgruppe sechs Teilnehmende mit besonderem Förderbedarf im FSJ (Thomas Bibisidis, Deutsches Rotes Kreuz). Diese können - ebenso wie die „IncomerInnen“ zusätzlich gefördert werden, um ihren besonderen Bedürfnissen besser gerecht zu werden. Arbeitsgruppe sieben (Jens Maedler, Bundesvereinigung kulturelle 
Kinder- und Jugendbildung) wiederum stellte Freiwillige und Einsatzstellen im ländlichen Räumen in den Mittelpunkt.

Die von Kristin Napieralla (BAK FSJ und Paritätischer Gesamtverband) dem damaligen Leiter sowie Axel-Kurt Schubert (BAFzA) moderierte und von Dr. Jens Kreuter, dem Leiter des Arbeitsstabes Freiwilligendienste im BMFSFJ, sowie Dr. Ralf-René Weingärtner, dem FSJ-Referatsleiter, begleitete Tagung bot Anlass für vielfältige Diskussionen. Einig waren sich alle Anwesenden in dem Bestreben, auch zukünftig neben der Seminararbeit auch die Bildungsprozesse in der Einsatzstelle sowie die pädagogische Begleitung durch die Träger wertzuschätzen und qualitativ hochwertig zu verwirklichen. 